\title{
Nahm's Transformation for Instantons
}

\author{
Peter J. BRAAM \\ Merton College \\ Oxford OX1 4.50 \\ United Kingdom \\ and \\ Pierre van $\mathrm{B} \Lambda \mathrm{AL}$ \\ CERN - Genewa
}

\begin{abstract}
We describe in mathematical detail the Nahm transformation which maps anti-sclf dual connections on the four-torus $\left(S^{1}\right)^{4}$ onto anti-self dual connections on the dual torus. This transformation induces a map betwen the relerant instanton moduli spaces and we show that this map is a (hyperKahlikr-) isometry.
\end{abstract}

CERN-TH.5108/88

July 1988 


\section{Introduction}

This paper deals with "magical" properties of U(n) anti-self-dual (asd) connections $A$ on a $C^{n}$ - bundle $F$ over a four-torus $T^{4}$. The "witchcraft" starts by introducing a family of Dirac operators coupled to $(F, A)$ parametrized by the dual torus $\hat{T}^{4}$. The families index turns out to be a bundle $\hat{F} \rightarrow \hat{T}^{4}$ (under a genericity assumption on $A$ ) and comes equipped with a natural connection $\hat{A}$, which is again asd (Theorem 1.5). This is Nahm's transform. Doing it again to $(\hat{F}, \hat{A})$, we obtain $(\hat{\hat{F}}, \hat{\hat{A}})$ and a unitary equivalence $(F, A) \sim(\hat{\hat{F}}, \hat{\hat{A}})$. In other words the square of Nahm's transform is the identity (Theorem 2.8\&2.9). This was discovered by the authors and independently by Schenk[21], and relies heavily on some ideas of Nahm[19,20]. The transformation now induces a map of moduli spaces of (generic) asd connections $N: \mathcal{M}(F) \rightarrow \mathcal{M}(\hat{F})$. The spaces $\mathcal{M}(F)$ and $\mathcal{M}(\hat{F})$ carry a hyperKähler metric and $N$ turns out to be a hyperKähler isometry , as was conjectured by S.K. Donaldson (Theorem 3.4).

This transformation has been around for a while, and the fact that its square is the identity was announced by Nahm[19,20] in the early eighties (see also Corrigan and Goddard[6]). However, for mathematicians it is not so easy to understand Nahm's work. In a way, the torus case treated here is the simplest version of Nahm's transform. Nahm originally developed his transformation for instantons invariant under subgroups of $R^{4}$, different from the 4-dimensional lattice (such as $R$ or $Z$ ). For time-invariant instantons this was used extensively by Hitchin[13] and Hurtubise-Murray[15] to study magnetic monopoles. In these cases the direct analytical attack is still missing.

Two further comments are in place. In algebraic geometry, Mukai[16] discovered the same transformation, but now in the category of coherent sheaves on abelian varieties. Mukai[18] applied this to the study of moduli spaces of stable bundles on abelian varieties and this could be of future use in mathematical physics. Antony Maciocia pointed out that one can use Mukai's work to give an alternative proof of the isometry property of the transform.

Secondly, it should be pointed out that Nahm's work is very reminiscent of certain methods used in the theory of completely integrable systems. Also there the solutions of an associated set of linear equations is a key ingredient, see e.g. Segal-Wilson[22] and Duistermaat-Grünbaum[12].

Both authors would like to thank Simon Donaldson,Antony Maciocia and Werner Nahm for discussions and the ITP at Stony Brook, CERN and Merton College for hospitality. P.J.B. is partially supported by a C\&C Huygens fellowship of the Netherlands Organization for Advancement of Science. 


\section{Connections on $T^{4}$ and the Nahm transform.}

Let $\Lambda \subset R^{4}$ be a lattice of rank 4. Then $T^{4}=R^{4} / \Lambda$ inherits from $R^{4}$ the structure of an oriented Riemannian manifold. Clearly $T^{4}$ is a four-dimensional torus, i.e. $T^{4}$ is diffeomorphic to $\left(S^{1}\right)^{4}$. We shall assume the volume to be 1. Let $\hat{R}^{4}$ denote the dual space of $R^{4}$ and define the dual lattice $\Lambda^{*}$ as

$$
\Lambda^{*}=\left\{\mu \in \hat{R}^{4} ; \mu(\lambda) \in Z, \forall \lambda \in \Lambda\right\}
$$

Cohomology classes on $T^{4}$ can be represented by constant, or equivalently, invariant differential forms, therefore

$$
H^{1}\left(T^{4} ; R\right)=\hat{R}^{4} \quad, \quad H^{1}\left(T^{4} ; Z\right)=\Lambda^{*}
$$

We can also define a dual torus $\hat{T}^{4}=\hat{R}^{4} / \Lambda^{*}$. Points in $\hat{T}^{4}$ parametrize the unitary flat connections on the trivial line bundle $T^{4} \times C=\mathcal{L} \rightarrow T^{4}$, up to gauge equivalence, because

$$
\operatorname{Repr}\left(\pi_{1}\left(T^{4}\right), S^{1}\right)=H^{1}\left(T^{4} ; R\right) / H^{1}\left(T^{4} ; Z\right)=\hat{T}^{4}
$$

Denote by $\pi: T^{4} \times \hat{R}^{4} \rightarrow T^{4}$ the projection. On the trivial line bundle $\pi^{*} \mathcal{L} \rightarrow T^{4} \times \hat{R}^{4}$ we have a universal connection 1 -form given by

$$
\omega(x, z)=2 \pi i \sum_{\mu=1}^{4} z_{\mu} d x_{\mu}
$$

where $x_{\mu}$ and $z_{\mu}$ are dual linear coordinates on $R^{4}$ and $\hat{R}^{4}$. Clearly $\omega(x, z)$ and $\omega(x, z+\lambda)$ are, for $\lambda \in \Lambda^{*}$, gauge equivalent connections on $T^{4}$; the gauge transformation $g(x)=\exp (-2 \pi i \lambda(x))$ satisfies $g \cdot \omega_{z}=\omega_{z+\lambda}$. This gives a quotient bundle $\mathcal{P} \rightarrow T^{4} \times \hat{T}^{4}$ with connection $\omega$. The bundle $\mathcal{P}$ is called the Poincaré bundle and the curvature of $\omega$ equals

$$
\Omega=2 \pi i \sum_{\mu} d z_{\mu} \wedge d x_{\mu}
$$

There is a canonical isomorphism $\hat{\hat{T}}^{4}=T^{4}$ and under this isomorphism $\hat{\mathcal{P}}=\mathcal{P}^{*}$, the dual of $\mathcal{P}$. In order to see this we use the gauge transformation $g(x, z)=\exp (-2 \pi i x . z)$ on $R^{4} \times \hat{R}^{4}$ to transform $\omega(x, z)$ into $-2 \pi i \sum_{\mu} x_{\mu} d z_{\mu}$, which is minus the universal connection on $R^{4} \times \hat{T}^{4}$.

Let $Q$ be a principal $\mathrm{U}(\mathrm{n})$-bundle over $T^{4}$ equiped with a connection $A$. Let $F=Q \times_{\mathrm{U}(\mathrm{n})} C^{n}$ and define a family of connections $A_{z}$ on $F$ by $A_{z}=A+2 \pi i I d_{n} \sum_{\mu} z_{\mu} d x_{\mu}$. Clearly $A_{z}$ is the connection $A \otimes 1+I d_{n} \otimes \omega$ on $\pi^{*}(F \otimes \mathcal{L})$ restricted to $T^{4} \times\{z\}$. Because the modification lies in the centre of $\mathrm{U}(\mathrm{n})$ the curvature of $A_{z}$ equals that of $A$. In the same way we can equip $\mathcal{P} \otimes \pi^{*} F$ with a connection $A_{z}$.

If $S^{+}, S^{-} \rightarrow T^{4}$ denote the spin-bundles of $T^{4}$ then we have Dirac operators:

$$
\begin{aligned}
& D_{A_{2}}^{+}: \Gamma\left(T^{4}, S^{+} \otimes F\right) \rightarrow \Gamma\left(T^{4}, S^{-} \otimes F\right) \\
& D_{A_{z}}^{-}: \Gamma\left(T^{4}, S^{-} \otimes F\right) \rightarrow \Gamma\left(T^{4}, S^{+} \otimes F\right)
\end{aligned}
$$


A connection $A$ on $F$ is said to be 1-irreducible if there are no covariantly constant $s \in \Gamma\left(T^{4}, F\right)$ for any $A_{z}, z \in \hat{T}^{4}$; this concept is due to Donaldson-Kronheimer[10]. If the holonomy of $A_{z}$ is an open set in $U(n)$ (for all $z$ ) then $A$ is certainly 1 -irreducible.

Lemma 1.1: If $A$ is 1-irreducible and anti-self dual then $\operatorname{ker} D_{A_{*}}^{+} \subset \Gamma\left(T^{4}, S^{+} \otimes F\right)$ is zero for all $z$.

Proof: Use the Weitzenboch formula:

$$
D_{A_{x}}^{-} D_{A_{*}}^{+}=\nabla_{A_{x}}^{*} \nabla_{A},
$$

see Atiyah-Hitchin-Singer[3], $\S 6$.

For 1-irreducible $A$ the vector spaces $\hat{F}_{z}=\operatorname{ker} D_{A_{z}}^{-}$build up a smooth vector bundle $\hat{F}$ over $\hat{T}^{4}$. Let $\hat{H} \rightarrow \hat{T}^{4}$ be the bundle of Hilbert spaces with fibre $\hat{H}_{z}=L^{2}\left(T^{4}, S^{-} \otimes F \otimes \mathcal{P}_{\left[T^{4} \times\{z\}\right.}\right)$. Then $\hat{F}$ is a subbundle of $\hat{H}$. Observe further that the "horizontal-component" $\frac{d}{d z}$ of the connection $A \otimes 1+I d_{n} \otimes \omega$ induces a (flat) connection $\hat{d}$ on $\hat{H} \rightarrow \hat{T}^{4}$, and that over $\hat{R}^{4}, \hat{\pi}^{*} \hat{F}$ is a Hermitian subbundle of the trivial, flat Hilbert space bundle with fibre $L^{2}\left(T^{4}, S^{-} \otimes F\right)$. This will be important for computational purposes. Let

$$
P: \hat{H} \rightarrow \hat{F}
$$

denote the $L^{2}-$ projection, then it is well known (see e.g. Atiyah[1]) that:

$$
\hat{\nabla}=P \hat{d}: \Gamma\left(\hat{T}^{4}, \hat{F}\right) \rightarrow \Gamma\left(\hat{T}^{4}, \Lambda^{1} \otimes \hat{F}\right)
$$

defines a connection $\hat{A}$ on $\hat{F}$.

Definition 1.2: The Nahm transform $\mathcal{N}$ of $(F, A)$ is the pair of vector bundle and connection $(\hat{F}, \hat{A})$.

First we shall study the topology of $\hat{F}$. It is not very hard to prove that, topologically, $U(\mathrm{n})$ bundles $F \rightarrow T^{4}$ are determined by

$$
n=\operatorname{rk}(F), c_{1}(F) \in H^{2}\left(T^{4}, Z\right), c_{2}(F) \in H^{4}\left(T^{4}, Z\right) .
$$

For $n>1$ and any $c_{1}(F), c_{2}(F)$ a bundle $F$ can be constructed, and if $F$ is a line bundle $(n=1)$ then $c_{2}(F)=0$ is the only constraint on these data. If one assumes that $F$ carries an anti-self dual connection then $c_{1}(F)$ must be an anti-self dual class in $H^{2}\left(T^{4}, R\right) \cap H^{2}\left(T^{4}, Z\right)$. For this the three dimensional eigenspace of $*$ in $H^{2}\left(T^{4}, R\right)$ with eigenvalue -1 , must have a non-empty intersection with the lattice $H^{2}\left(T^{4}, Z\right)$. This depends on the Riemannian structure, and for a generic flat torus the only integral anti-self dual class is 0 . Concerning the topological invariants of $\hat{F}$ we have:

Proposition 1.3: Let $A$ be a 1-irreducible asd conection on $F$. For $\hat{F}$ we have

$$
\begin{aligned}
\operatorname{rk}(\hat{F}) & =-\frac{1}{2} p_{1}\left(F_{R}\right)=c_{2}(F)-\frac{1}{2} c_{1}^{2}(F) \\
c_{1}(\hat{F}) & =\frac{1}{4 \pi^{2}} \Omega \cup \Omega \cup c_{1}(F) /\left[T^{4}\right] \\
p_{1}\left(\hat{F}_{R}\right) & =-2 \operatorname{rk}(F) \quad \text { or } \quad c_{2}(\hat{F})=\operatorname{rk}(F)+\frac{1}{2} c_{1}^{2}(F),
\end{aligned}
$$


Here / denotes slant product, i.e. integration over $T^{4}, U$ denotes cup product and $\left(F_{R}, \hat{F}_{R}\right)$ are the underlying real bundles of $(F, \hat{F})$.

Proof: The Atiyah-Singer index theorem for families (see Atiyah-Singer[4]) asserts that

$$
\operatorname{ch}(\hat{F})=\operatorname{rk}(\hat{F})+c_{1}(\hat{F})+\frac{1}{2} p_{1}(\hat{F})=-\operatorname{ch}(\mathcal{P}) \cup \operatorname{ch}(F) \cup \hat{\mathcal{A}}\left(T^{4}\right) /\left[T^{4}\right],
$$

with $\hat{\mathcal{A}}(X)$ the $\hat{\mathcal{A}}$ - genus of $X$. This proves the proposition.

This proposition has a nice corollary (see Mukai[16],[18] and also Schenk[21]):

Corollary 1.4: There exist no asd connctions $A$ on a bundle $F$ with $c_{1}(F)=0, c_{2}(F)=1$.

Proof: It is easy to see that such a pair $(F, A)$ would be 1 -irreducible. Thus $\hat{F}$ is a vector bundle of rank 1 with $c_{2} \neq 0$, which is impossible.

If one allows a twist such 1-instantons do exist. More precisely let $Q \rightarrow T^{4}$ be an $\mathrm{SO}(3)$ bundle with non-zero second Stiefel-Witney class $\left(w_{2}(Q) \neq 0\right.$, i.e. there is twist). Such a bundle has a unique flat connection $A_{o}$, upto SO(3)-gauge transformations for which all cohomology groups of the Atiyah-HitchinSinger complex [3],\$6 vanish. Using the construction of Taubes[23] and Donaldson[11] one can "attach" a localized 1-instanton from $S^{4}$ to this flat connection in order to obtain a new asd connection on a bundle $Q^{\prime} \rightarrow T^{4}$ with $w_{2}\left(Q^{\prime}\right)=w_{2}(Q)$ and $p_{1}\left(Q^{\prime}\right)=-4$. The dimension of the hyperKähler moduli space $\mathcal{M}$ of these connections is 8 . The eight parameters asymptotically equal centre and scale of the attached, localized instanton $\left(T^{4} \times(0, \epsilon)\right)$ and finally the attaching parameters in $\mathrm{SO}(3) / Z_{2}$, where $Z_{2}$ is the stabilizer of $A_{o}$. Consequently $\mathcal{M}$ modulo the 4-torus, acting by translations, is a smooth hyperKähler 4-manifold, which is presumably related to a K3-surface.

The Nahm transformation has a "magic" property:

Theorem 1.5: Let $A$ be asd and 1-irreducible then $\hat{A}$ is asd on $\hat{F}$.

Proof: It will be expedient to introduce a more detailed notation. Let $\hat{f}^{j}(z)=\psi_{z}^{j}(x) \in L^{2}\left(T^{4}, S^{-} \otimes \mathcal{L}_{z} \otimes F\right)$ with $z \in \hat{R}^{4}, j=1, \cdots,-\frac{1}{2} p_{1}(F)$ be an orthonormal framing of $\hat{F}$ on $\hat{R}^{4}$. Note that $\mathcal{L}$ is trivialized on $T^{4} \times \hat{R}^{4}$, using the connection $\omega=2 \pi i z_{\mu} d x_{\mu}$, therefore we map into the fixed vector space $L^{2}\left(T^{4}, S^{-} \otimes \mathcal{L}_{0} \otimes F\right)$, which is isomorphic to $L^{2}\left(T^{4}, S^{-} \otimes F\right)$.

Then for a section $\hat{\boldsymbol{s}}(z)=\sum_{j} \hat{s}_{j}(z) \hat{f}^{j}(z), \hat{s}_{j} \in C^{\infty}\left(\hat{R}^{4}\right)$ we have (with $P: L^{2}\left(T^{4}, S^{-} \otimes F\right) \otimes \hat{\Lambda}^{1} \rightarrow \hat{F} \otimes \hat{\Lambda}^{1}$ the projection):

$$
\hat{\nabla} \hat{s}=P \hat{d} \hat{s}=\left(1-D_{A_{z}}^{+} G_{z} D_{A_{z}}^{-}\right)\left[\hat{d}\left(\hat{s}_{j}(z) \psi_{z}^{j}(x)\right)\right],
$$

because, using Hodge theory,

$$
P=\left(1-D_{A_{*}}^{+} G_{z} D_{A_{*}}^{-}\right)
$$

with $G_{z}=\left(D_{A_{z}}^{-} D_{A_{z}}^{+}\right)^{-1}$ acting on $L^{2}\left(T^{4}, F \otimes S^{-}\right)$. For $P$ we also have a pedestrian expression. If $r \in$ $L^{2}\left(T^{4}, F \otimes S^{-}\right)$then

$$
P r=\sum_{j}<\hat{f}^{j}, r>\hat{f}^{j},
$$


where $<,>$ denotes the $L^{2}\left(T^{4}, F \otimes S^{-}\right)$inner product. So

$$
\hat{\nabla} \hat{s}=\left(\hat{d} \hat{s}_{j}+\hat{A}_{j k} \hat{s}_{k}\right) \hat{f}^{j}
$$

with $\hat{A}_{j k}$ the connection matrix of $\hat{A}$ equal to

$$
\hat{A}_{j k}=\left\langle\hat{f}^{j}, \hat{d} \hat{f}^{k}\right\rangle=\left\langle\psi_{z}^{j}, \hat{d} \psi_{z}^{k}\right\rangle
$$

For the curvature one easily finds:

$$
\begin{aligned}
\hat{F}_{i j} & =\hat{d} \hat{A}_{i j}+\hat{A}_{i k} \wedge \hat{A}_{k j} \\
& =<\hat{d} \psi_{z}^{i}, \wedge \hat{d} \psi_{z}^{j}>+<\psi_{z}^{i}, \hat{d} \psi_{z}^{k}>\wedge<\psi_{z}^{k}, \hat{d} \psi_{z}^{j}>
\end{aligned}
$$

Now $\left\langle\hat{d} \psi_{z}^{i}, \psi_{z}^{k}>=-\left\langle\psi_{z}^{i}, \hat{d} \psi_{z}^{k}\right\rangle\right.$, so

$$
\begin{aligned}
\hat{F}_{i j} & =<\hat{d} \psi_{z}^{i}, \wedge \hat{d} \psi_{z}^{j}>-<P \hat{d} \psi_{z}^{i}, \wedge \hat{d} \psi_{z}^{j}> \\
& =<D_{A_{z}}^{+} G_{z} D_{A_{z}}^{-} \hat{d} \psi_{z}^{i}, \wedge \hat{d} \psi_{z}^{j}> \\
& =<G_{z} D_{A_{z}}^{-} \hat{d} \psi_{z}^{i}, \wedge D_{A_{z}}^{-} \hat{d} \psi_{z}^{j}>.
\end{aligned}
$$

But

$$
D_{A_{z}}^{-} \hat{d} \psi_{z}^{i}=\left[D_{A_{z}}^{-}, \hat{d}\right] \psi_{z}^{i}=-\Omega \cdot \psi_{z}^{i} .
$$

The dot in this equation stands for Clifford multiplication, using the fact that there is a covariantly constant framing of $S^{+}, S^{-}$and $T^{*} T^{4}$. Thus $G_{z}$ acts on $L^{2}\left(T^{4}, S^{+} \otimes F\right) \simeq S^{+} \otimes_{C} L^{2}\left(T^{4}, F\right)$ as $I d_{S+} \otimes_{C} \mathcal{G}_{z}$ with $\mathcal{G}_{z}=\left(\nabla_{A_{z}}^{*} \nabla_{A_{4}}\right)^{-1}$ acting on $L^{2}\left(T^{4}, F\right)$. This shows that $G_{z}$ commutes with Clifford multiplication. Thus we obtain

$$
\left.\hat{F}_{i j}=-<\Omega \wedge \Omega \cdot \psi_{z}^{i}, G_{z} \psi_{z}^{j}\right\rangle
$$

with $\Omega \wedge \Omega \cdot \psi_{z}^{i}=(2 \pi)^{2} \sum_{\mu, \nu} d z_{\mu} \wedge d z_{\nu} d x_{\mu} \cdot d x_{\nu} \cdot \psi_{z}^{i}$ which is an element of $\hat{\Lambda}_{-}^{2} \otimes S^{-} \otimes F$

The formal manipulations in this proof will appear again and will sometimes be left to the reader.

There is a holomorphic version of the Nahm transformation, which has been discovered by Mukai[16],[18]. For this one needs to choose a holomorphic structure on $T^{4}$. The Poincare bundle now appears as a holomorphic line bundle on $T^{4} \times \mathrm{Pic}^{\circ}\left(T^{4}\right)$; here $\mathrm{Pic}^{\circ}\left(T^{4}\right) \simeq \hat{T}^{4}$ in a canonical way. A bundle $F$ carrying a 1-irreducible asd connection is holomorphic and stable; conversely every stable holomorphic bundle carries a unique asd connection inducing the holomorphic structure, see Donaldson[8]. Mukai's "Fourier functor" is defined as:

$$
\mathcal{F}(F)=R^{1} \hat{\pi}_{*}\left(\mathcal{P} \otimes \pi^{*} F\right)
$$

where $\hat{\pi}: T^{4} \times \hat{T}^{4} \rightarrow \hat{T}^{4}$ is the projection. If $F$ is a stable vector bundle then also $\mathcal{F}(F)$ will be stable and we shall prove (see also Donaldson-Kronheimer[10]) that $\mathcal{F}(F)$ is the holomorphic bundle underlying $(\hat{F}, \hat{A})$. 
In fact the fibre $\mathcal{F}(F)_{z},\left(z \in \hat{T}^{4}\right)$ equals $H^{1}\left(T^{4}, F \otimes L\right)$, where $L=\left.\mathcal{P}\right|_{T^{4} \times z}$. By Hodge theory this space is canonically isomorphic to the kernel of $\bar{\partial}_{F \otimes L} \oplus \bar{\partial}_{F \otimes L}^{*}: \Gamma\left(T^{4}, \Lambda^{0,1} \otimes F \otimes L\right) \rightarrow \Gamma\left(T^{4},\left(\Lambda^{0}+\Lambda^{0,2}\right) \otimes F \otimes L\right)$. However, this operator is precisely the Dirac operator $D_{A}^{-}$, discussed earlier (see Atiyah[2]). This establishes an isomorphism $\mathcal{F}(F) \rightarrow \hat{F}$ as bundles. It remains to show that the holomorphic structure on $\mathcal{F}(F)$ coincides with that of $\hat{F}$. This was proved in a general context by Bismut-Gillet-Soulé[7] (Proposition 3.10 \& Theorem 3.11 ).

In order to prove our next proposition we need the quaternionic structure on spinors on 4-manifolds. There is an anti-linear, covariantly constant map

$$
\varepsilon: S^{ \pm} \rightarrow S^{ \pm}
$$

satisfying $\varepsilon^{2}=-1$. Tensoring this with the anti-linear map: $E \rightarrow E^{*}$ for any vector bundle $E$ gives an anti-linear map $\hat{\varepsilon}: S^{ \pm} \otimes E \rightarrow S^{ \pm} \otimes E^{*}$ which still commutes with Clifford multiplication.

Proposition 1.6: Assume that $(F, A)$ is asd and 1-irreducible. Then $\mathcal{N}\left(F^{*}, A^{*}\right)$ is isomorphic to $\left((-1)^{*} \hat{F}^{*},(-1)^{*} \hat{A}^{*}\right)$. Here $A^{*}$ denotes the connection on the dual bundle $F^{*}$ of $F$, and -1 is the inversion map on $\hat{T}^{4}$.

Proof: Let $\psi_{z} \in L^{2}\left(T^{4}, F \otimes \mathcal{L}_{z} \otimes S^{-}\right)$be a harmonic spinor for $D_{A_{*}}^{-}$. Then $\hat{\varepsilon}\left(\psi_{z}\right) \in L^{2}\left(T^{4}, F^{*} \otimes \mathcal{L}_{-z} \otimes S^{-}\right)$ is a harmonic spinor for $D_{A_{-}^{*}}^{-}$. Indeed, let $e_{\mu}$ be a covariantly constant orthonormal framing of $T^{*} T^{4}$ then:

$$
\begin{aligned}
D_{A_{-s}^{-}}^{-}\left(\hat{\varepsilon} \psi_{z}\right) & =e_{\mu} \cdot\left(\frac{\partial}{\partial x_{\mu}}\left(\hat{\varepsilon} \psi_{z}\right)-A_{\mu}^{\mathrm{t}} \hat{\varepsilon} \psi_{z}-2 \pi i z_{\mu} \hat{\varepsilon} \psi_{z}\right) \\
& =e_{\mu} \cdot \hat{\varepsilon}\left(\frac{\partial}{\partial x_{\mu}}+A_{\mu} \psi_{z}+2 \pi i z_{\mu} \psi_{z}\right) \\
& =\hat{\varepsilon}\left(e_{\mu} \cdot \nabla_{A_{z}}^{\mu} \psi_{z}\right)=\hat{\varepsilon}\left(D_{A_{z}}^{-} \psi_{z}\right)=0 .
\end{aligned}
$$

Therefore we have a complex linear bundle map $(-1)^{*} \hat{F}^{*} \rightarrow \mathcal{N}\left(F^{*}\right)$. It is trivial to show that this preserves connections.

In Mukai[16] the reader can also find a convolution type relation between $\mathcal{F}(E \otimes F)$ and $\mathcal{F}(E) \otimes \mathcal{F}(F)$, for two vector bundles $E$ and $F$.

We end this section with a remark of a different nature. The Nahm transformation can be studied more generally for asd connections $A$ on $R^{4}$, invariant under a subgroup of translations $\Lambda \subset R^{4}$. Possible choices of $\Lambda$ include $\Lambda=\{0\}$, these are the ordinary instantons on $S^{4} . \Lambda=R$ gives rise to monopoles, whereas $\Lambda=Z$ will correspond to the so-called calorons. In this paper, we are restricting ourselves to instantons on the 4-torus, for which $\Lambda=Z^{4}$. For the more generalized situations mentioned above, going through a construction similar to ours, we see that the Nahm transformation gives rise to new instantons on $\hat{R}^{4}$, which are invariant under

$$
\Lambda^{*}=\left\{\alpha \in \hat{R}^{4} ; \alpha(\lambda) \in Z, \forall \lambda \in \Lambda\right\} .
$$

If $\Lambda=\{0\}$ this is closely related to the celebrated ADHM construction, see Nahm[20] and DonaldsonKronheimer[10]. In the case of magnetic monopoles $(\Lambda=R)$, Nahm's transformation has been a powerful tool 
to understand moduli spaces, see Hitchin[13], Donaldson[9], Hurtubise-Murray[15] and Atiyah-Hitchin[5]. In a forthcoming paper we shall consider a Nahm transformation for instantons on $T^{3} \times R$, which can be studied as monopoles on $T^{3}$ with some singularities. Instantons on $T^{3} \times R$ are intimately related to problems of confinement, see 't Hooft[14].

\section{The square of Nahm's transform}

In this section we shall prove that there is a canonical isomorphism $(F, A) \simeq(\hat{\hat{F}}, \hat{\hat{A}})$ of Hermitian bundles with asd connections. Before starting in honest, we give an outline of the ingredients of the proof.

a. We express harmonic spinors for $\hat{D}_{\hat{A}_{x}}^{-}$in terms of those for $D_{A_{x}}^{-}$, using the Green operator $G_{z}$.

b. It is shown that $\hat{A}$ is 1-irreducible, by explicitly giving the inverse $\hat{G}_{x}$ of $\hat{\Delta}_{\hat{A}_{x}}$

c. To prove that the spinors found in a. form an orthonormal framing of $\hat{\hat{F}}$ we need $\hat{F} \otimes S^{-}$-innerproducts of spinors for $\hat{D}_{\hat{A}_{x}}$. These appear as the Laplacian in $z$ applied to the $z$-dependent operator $G_{z}$. Here we use the first term of the celebrated expansion of $G_{z}\left(x_{1}, x_{2}\right)$.

d. Finally we can compare $A$ and $\hat{\hat{A}}$ using the same method and the second term in the expansion.

Recall that $\hat{F}$ was defined as a subbundle of $\hat{H} \rightarrow \hat{T}^{4}$. So we have a canonical element:

$$
\Psi \in \Gamma\left(T^{4} \times \hat{T}^{4}, \hat{\pi}^{*} \hat{F}^{*} \otimes \pi^{*} F \otimes S^{-} \otimes \mathcal{P}\right)
$$

such that $\Psi(\hat{f})\left(\hat{f} \in \hat{F}_{z}\right)$ is annihilated by $D_{A_{z}}^{-}$on $T^{4} \times\{z\}$. If $G_{z}=\left(D_{A_{z}}^{-} D_{A_{z}}^{+}\right)^{-1}$ then tensoring over $C^{\infty}\left(\hat{T}^{4}\right)$ with $i d_{\hat{F}}$ we obtain a section:

$$
G \Psi \in \Gamma\left(T^{4} \times \hat{T}^{4}, \hat{\pi}^{*} \hat{F}^{*} \otimes \pi^{*} F \otimes S^{-} \otimes \mathcal{P}\right)
$$

We shall use the metric to let $T T^{4} \simeq T^{*} \hat{T}^{4}$ act on $S^{ \pm}$, thereby identifying $S^{ \pm}$with $\hat{S}^{ \pm}$.

Proposition 2.1: For $f \in F_{x}^{*}$ the section $G \Psi(f) \in \Gamma\left(\{x\} \times \hat{T}^{4}, \hat{\pi}^{*} \hat{F}^{*} \otimes S^{-} \otimes \mathcal{P}\right)$ lies in $\operatorname{ker} \hat{D}_{\hat{A}^{*}}^{-}$.

Proof: First we will state the following

Lemma 2.2: $G_{z} \nabla_{A_{s}}^{\mu} G_{z}=\frac{1}{4 \pi i} \frac{\partial G_{z}}{\partial z_{\mu}}$ where $x_{\mu}, z_{\mu}$ are dual linear coordinates on $R^{4}, \hat{R}^{4}$ and $\nabla_{A_{s}}^{\mu} s=$ $\nabla_{A_{*}}(s)\left(\frac{\partial}{\partial x_{\mu}}\right)$.

Proof of the lemma follows from $\left[\nabla_{A_{s}}^{*} \nabla_{A_{*}}, \frac{\partial}{\partial z_{\mu}}\right]=4 \pi i \nabla_{A_{s}}^{\mu}$.

As before, let a local framing $\hat{f}^{j}(z)$ of $\hat{F}$ be given, together with the framing of $\mathcal{P}$ on $T^{4} \times \hat{R}^{4}$. Then $\hat{R}^{4} \ni z \rightarrow \Psi\left(\hat{f}^{j}(z)\right)=\psi_{z}^{j} \in \Gamma\left(T^{4}, F \otimes \mathcal{L} \otimes S^{-}\right)$and $(G \Psi)\left(\hat{f}^{j}\right)=G_{z} \psi_{z}^{j}$. If $f \in F_{x}^{*}$ and $\hat{f}_{j}^{*}$ is the framing of $\hat{F}^{*}$ dual to $\hat{f}^{j}$ then $(G \Psi)(f)=\sum_{j}\left(G_{z} \psi_{z}^{j}\right)(f) \hat{f}_{j}^{*}$, and now we have the connection matrix $\hat{A}_{i j}^{*}=-\hat{A}_{i j}^{t}$ for $\hat{F}^{*}$ at our disposal (observe that in this trivialization the connection on $\mathcal{P}$ has no $d z$ components). Let $e_{\mu}, \hat{e}_{\mu}$ be dual orthonormal framings of $T T^{4}, T \hat{T}^{4}$ which can be identified, using the metric. Following AtiyahHitchin-Singer[3] we choose the relations in the Clifford algebra such that $e_{\mu} e_{\nu}+e_{\nu} e_{\mu}=-2 \delta_{\mu \nu}$, so the inner products on $S^{ \pm}$satisfy $\left.\left\langle e_{\mu} s_{+}, s_{-}\right\rangle_{S^{-}}=-<s_{+}, e_{\mu} s_{-}\right\rangle_{S_{+}}$. Then we have

$$
\begin{aligned}
\left(\hat{D}_{\hat{\hat{A}}}^{-}(G \Psi)(f)\right)^{i} & \left.=\hat{e}_{\mu} \cdot\left\{\delta_{i, j} \frac{\partial}{\partial z_{\mu}}-<\psi_{z}^{j}, \frac{\partial}{\partial z_{\mu}} \psi_{z}^{i}>\right\}\left(G_{z} \psi_{z}^{j}\right)(f)\right) \\
& =\hat{e}_{\mu} \cdot\left\{\frac{\partial}{\partial z_{\mu}}\left(G_{z} \psi_{z}^{i}\right)(f)-G_{z}\left(1-D_{A_{z}}^{+} G_{z} D_{A_{z}}^{-}\right) \frac{\partial \psi_{z}^{i}}{\partial z_{\mu}}(f)\right\}
\end{aligned}
$$


Using Lemma $2.2, D_{A,}^{+}=e_{\mu} \nabla_{A_{*}}^{\mu}$ and $D_{A_{s}}^{-} \frac{\partial \psi_{x}^{j}}{\partial z_{\mu}}=-2 \pi i e_{\mu} \psi_{z}^{j}$ this equals:

$$
\hat{e}_{\mu} \cdot\left(\delta_{\mu, \nu}-\frac{1}{2} e_{\nu} e_{\mu}\right)\left(\frac{\partial G_{z}}{\partial z_{\nu}} \psi_{z}^{i}\right)(f)
$$

which is easily seen to vanish, using $\sum_{\mu} e_{\mu} e_{\nu} e_{\mu}=2 e_{\nu}$

This is quite a remarkable formula as it relates the spinors for $A$ with those for $\hat{A}$, connections on bundles over different manifolds! In homological algebra such results can be obtained by using spectral sequences or, in a more sophisticated version, derived categories. Donaldson-Kronheimer[10] and Mukai[16] proceed in this way.

Reasoning as in the proof of Proposition 1.6 one sees that

$$
u=4 \pi \hat{\varepsilon}(G \Psi)
$$

is a map from $F_{x}$ to the harmonic spinors for $\hat{D}_{\hat{A}}^{-}$acting on $\Gamma\left(\hat{T}^{4}, \hat{\mathcal{P}}_{x} \otimes S^{-} \otimes \hat{F}\right)$. We shall now show that it is actually an isometry $F \rightarrow \hat{\hat{F}}$. Our reasoning closely follows the exposition given by $S \operatorname{chenk}$ [21].

First we shall establish that $(\hat{F}, \hat{A})$ is 1-irreducible, by showing that $\hat{\nabla}_{\hat{A}}^{*} \hat{\nabla}_{\hat{A}}$ has an inverse. The inverse can actually be written down quite explicitly. Recall that the maps $\hat{R}^{4} \rightarrow L^{2}\left(T^{4}, S^{-} \otimes F\right): z \rightarrow \psi_{z}^{j}=$ $\Psi\left(\hat{f}^{j}(z)\right)$ describe a section of $\hat{\pi}^{*} \hat{F}^{*} \otimes \pi^{*} F \otimes S^{-} \otimes \mathcal{P}$ over $T^{4} \times \hat{T}^{4}$. Assume that for $\lambda \in \Lambda^{*}, \lambda \rightarrow \hat{g}_{\lambda}(z)_{i j}$ is a set of automorphy factors for $\hat{F}$ then we may therefore assume that on $\hat{R}^{4}$ :

$$
\psi_{z+\lambda}^{i}(x)=\exp (-2 \pi i \lambda(x)) \hat{g}_{\lambda}(z)_{i j} \psi_{z}^{j}(x)
$$

Sections of $\hat{F}$ satisfy similar relations with $\exp (-2 \pi i \lambda(x))$ left out. Thus the object:

$$
\hat{G}\left(z_{1}, z_{2}\right)_{i j}=\frac{1}{4 \pi^{2}} \sum_{\mu \in \hat{\Lambda}} \int_{T^{4}} d x \exp (2 \pi i \mu(x)) \frac{<\psi_{z_{1}}^{i}(x), \psi_{z_{2}}^{j}(x)>}{\left|\mu-z_{1}+z_{2}\right|^{2}}
$$

represents for $z_{1}-z_{2} \notin \Lambda^{*}$ an element of $\hat{F}_{z_{1}} \otimes \hat{F}_{z_{2}}^{*}$. Here $<,>$ is the Hermitian product on $F \otimes S^{-}$, which is antilinear in the first argument so as to correctly give the linear map $\hat{G}: \hat{F}_{z_{2}} \rightarrow \hat{F}_{z_{1}}$. Observe that the summation is over Fourier coefficients of a smooth function. These decrease rapidly and this ensures locally uniform convergence of all derivatives in the summation.

In order to prove that $\hat{G}$ is the inverse of $\hat{\Delta}_{\hat{A}} \equiv \hat{\nabla}_{\hat{A}}^{*} \hat{\nabla}_{\hat{A}}$, we will need two lemmas. We shall encounter sections of $\hat{F}$ of the form $\sum_{j}\left\langle\psi_{z}^{j}, s\right\rangle \hat{f}^{j}(z)$, where $s \in L^{2}\left(T^{4}, S^{-} \otimes F\right)$. The following two lemmas give information about covariant derivatives of these sections.

Lemma 2.3:

$$
\left(\hat{\nabla}_{\hat{A}^{*}}^{\mu}\right)_{i j} \psi_{z}^{j}=-2 \pi i D_{A_{z}}^{+} G_{z} e_{\mu} \psi_{z}^{i}
$$

Equivalently one has for arbitrary

$s \in L^{2}\left(T^{4}, F \otimes S^{-}\right)$

$$
\left(\hat{\nabla}_{\hat{A}}^{\mu}\right)_{i j}\left\langle\psi_{z}^{j}, s>=-2 \pi i<\psi_{z}^{i}, G_{z} e_{\mu} D_{A_{z}}^{-} s\right\rangle
$$


where the inner product is for $L^{2}\left(T^{4}, F \otimes S^{-}\right)$.

Proof: Substitute $\left(\hat{\nabla}_{\hat{A}^{*}}^{\mu}\right)_{i j}=\delta_{i j} \frac{\partial}{\partial z_{\mu}}-<\psi_{z}^{j}, \frac{\partial \psi_{i}^{i}}{\partial z_{\mu}}>$ and proceed as in the proof of Proposition 2.1.

The next lemma will also play an important role in section 3 .

Lemma 2.4:

$$
\left(\hat{\triangle}_{\hat{A}^{*}}\right)_{i j} \psi_{z}^{j}=(4 \pi)^{2} G_{z} \psi_{z}^{i}
$$

or equivalently for any $s \in L^{2}\left(T^{4}, F \otimes S^{-}\right)$

$$
\left(\hat{\triangle}_{\hat{A}}\right)_{i j}\left\langle\psi_{z}^{j}, s\right\rangle=(4 \pi)^{2}\left\langle\psi_{z}^{i}, G_{z} s\right\rangle
$$

Proof: Employing the previous lemma, we can write

$$
\begin{aligned}
\left(\hat{\Delta}_{\hat{A}^{*}}\right)_{i j} \psi_{z}^{j} & =\left(\hat{\nabla}_{\hat{A}^{*}}\right)_{i k}\left(2 \pi i D_{A_{z}}^{+} G_{z} e_{\mu} \psi_{z}^{k}\right) \\
& =\frac{\partial}{\partial z_{\mu}}\left(2 \pi i D_{A_{z}}^{+} G_{z} e_{\mu} \psi_{z}^{i}\right)-2 \pi i D_{A_{z}}^{+} G_{z} e_{\mu} P \frac{\partial \psi_{z}^{i}}{\partial z_{\mu}}
\end{aligned}
$$

Using $\left[\frac{\partial}{\partial z_{\mu}}, D_{A_{s}}^{+}\right]=2 \pi i e_{\mu}$ and Lemma 2.2, together with the by now standard manipulations will finish the proof.

Proposition 2.5: $\hat{G}$ is the integral kernel of the inverse of $\hat{\Delta}_{\hat{\boldsymbol{A}}}$. Therefore $(\hat{F}, \hat{A})$ is 1-irreducible.

Proof: Assume $\hat{\triangle}_{\hat{A}}$ acts on the $z_{1}$ variable. First we show $\left(\hat{\Delta}_{\hat{A}} \hat{G}\right)\left(z_{1}, z_{2}\right)=0$ if $z_{1}-z_{2} \notin \Lambda^{*}$. This goes by brute force: Applying Lemma 2.3 to the section $s(x)=\exp (2 \pi i \mu(x)) \psi_{z_{2}}^{j}(x)$ gives

$$
\left(\hat{\nabla}_{\hat{A}}^{\alpha}\right)_{i k}<\psi_{z_{1}}^{k}, s>=(2 \pi)^{2}\left(\mu-z_{1}+z_{2}\right)_{\alpha}<\psi_{z_{1}}^{i}, G_{z_{1}} \psi_{z_{2}}^{j}>
$$

and combining with the result of Lemma 2.4

$$
\left(\hat{\triangle}_{\hat{A}}\right)_{i k} \int_{T^{4}} d x \exp (2 \pi i \mu(x))\left|\mu-z_{1}+z_{2}\right|^{-2}<\psi_{z_{1}}^{k}, \psi_{z_{2}}^{j}>
$$

gives

$$
\int_{T^{*}} d x \exp (2 \pi i \mu(x))<\psi_{z_{1}}^{i}, \psi_{z_{2}}^{j}>\hat{d}^{*} \hat{d}\left|\mu-z_{1}+z_{2}\right|^{-2}
$$

which is zero for $z_{1}-z_{2} \notin \Lambda^{*}$. This shows that $\int_{T^{*}} d z_{1} \hat{G}\left(z_{1}, z_{2}\right)\left(\hat{\Delta}_{\hat{A}} f\right)\left(z_{1}\right)=0$ for $f$ supported in $\hat{T}^{4}-\left\{z_{2}\right\}$. To finish the proof one simply has to observe three points:

Near $z_{2}$

a) $\hat{\Delta}_{\hat{A}}=\hat{d}^{*} \hat{d}+$ something bounded.

b) $\hat{G}\left(z_{1}, z_{2}\right)=\frac{I d}{4 \pi^{2}\left|z_{1}-z_{2}\right|^{2}}+R_{z_{2}}\left(z_{1}\right)$ with $\hat{\Delta}_{\hat{A}} R_{z_{2}}$ integrable.

c) $\frac{I d}{4 \pi^{2}\left|z_{1}-z_{2}\right|^{2}}$ is the Green's function for $\hat{d}^{*} \hat{d}$.

These three points are easy to see and quickly lead to a proof of the Green's function formula. The 1irreducibility follows from the fact that $\hat{A}$ is asd. 
We shall now show that $F_{x}^{*} \rightarrow L^{2}\left(\hat{T}^{4}, \hat{F}^{*} \otimes \mathcal{P}_{\mid\{x\} \times \hat{T}^{*}} \otimes S^{-}\right): f \rightarrow 4 \pi G \Psi(f)$ is an injective isometry. To study Nahm's transform on $F$ we used $\psi_{z}^{j}(x)$, which mapped $\hat{F}_{z}$ into the fixed vector space $L^{2}\left(T^{4}, S^{-} \otimes F\right)$, see the proof of Theorem 1.5. We now have to map $F_{x}$ into the fixed vector space $L^{2}\left(\hat{T}^{4}, S^{-} \otimes \hat{F}\right)$. This requires a trivialisation of the pullback of $\mathcal{P}$ over $R^{4} \times \hat{T}^{4}$, and this was discussed in section 1 . It follows that

$$
x \rightarrow v_{x}(z)=\exp (-2 \pi i z(x)) \hat{\varepsilon}\left(G_{z} \psi_{z}^{j}(x)\right) \hat{f}^{j}(z)
$$

describes a map $F_{x} \rightarrow L^{2}\left(\hat{T}^{4}, \hat{F} \otimes S^{-}\right)$. In this trivialisation the connection of the pullback of $\hat{\mathcal{P}} \otimes \pi^{*} \hat{F}$ over $R^{4} \times \hat{T}^{4}$ equals: $\hat{A}_{x}=\hat{A}+2 \pi i x_{\mu} d z_{\mu}$.

Just as $\left\langle\psi_{z_{1}}(x), \psi_{z_{2}}(x)\right\rangle_{F \otimes S-}$ gives a map $\hat{F}_{z_{3}} \rightarrow \hat{F}_{z_{1}}$, we can likewise use $\left\langle v_{x_{1}}(z), v_{x_{2}}(z)\right\rangle_{\hat{F} \bullet \otimes S^{-}}$as a map $F_{x_{2}} \rightarrow F_{x_{1}}$. We have

Lemma 2.6: $\left\langle v_{x_{1}}(z), v_{x_{2}}(z)\right\rangle_{\hat{F}^{*} \otimes S^{-}}=\exp \left(2 \pi i z\left(x_{1}-x_{2}\right)\right) \hat{d}^{*} \hat{d} G_{z}\left(x_{1}, x_{2}\right)$.

Proof: Since $v_{x}\left(\hat{f}_{j}^{*}(z)\right)=4 \pi \exp (-2 \pi i z(x)) \hat{\varepsilon}\left\{\int_{T^{4}} d y G_{z}(x, y) \psi_{z}^{j}(y)\right\}$ and $\left\langle\psi_{z}^{j}, f\right\rangle_{L^{2}\left(T^{4}, S-\otimes F\right)} \psi_{z}^{j}=P f$ we find that

$$
\left\langle v_{x_{1}}(z), v_{x_{2}}(z)\right\rangle_{\hat{F}_{*}^{*}}=(4 \pi)^{2} \exp \left(2 \pi i z\left(x_{1}-x_{2}\right)\right) G_{z} P G_{z}
$$

acting on $\Gamma\left(T^{4}, S_{-} \otimes F\right)$. As explained in the proof of Theorem 1.5 this operator is really a constant matrix acting on $S^{-}$, tensored over $C$ with a pseudo-differential operator acting on $L^{2}\left(T^{4}, F\right)$. Thus it makes sense to take the spinor $\operatorname{trace}^{\operatorname{tr}_{S}-}$ of eq.(2.4) which gives:

$$
<v_{x_{1}}(z), v_{x_{2}}(z)>_{S^{-} \otimes \hat{F}^{*}}=8 \pi^{2} \exp \left(2 \pi i z\left(x_{1}-x_{2}\right)\right)\left(\mathcal{G}_{z}^{2}+\mathcal{G}_{z} \nabla_{A_{z}}^{\mu} \mathcal{G}_{z} \nabla_{A_{z}}^{\mu} \mathcal{G}_{z}\right)
$$

acting on $L^{2}\left(T^{4}, F\right)$. A repeated application of Lemma 2.2 gives the required result.

That the map $u: F \rightarrow \hat{\hat{F}}$ is an isometry follows from:

Proposition 2.7 For all $x \in T^{4}$

$$
\int_{\hat{T}^{4}} d z<u_{x}^{i}(z), u_{x}^{j}(z)>_{\hat{F} \otimes S^{-}}=\delta_{i j}
$$

with $u_{x}^{j}(z)=u\left(f^{j}(x)\right)$ for $f^{j}(x)$ an orthonormal framing of $F_{x}$.

Proof: We obviously have:

$$
\left.<u_{x}^{i}(z), u_{x}^{j}(z)\right\rangle_{\hat{F} \otimes S^{-}}=\lim _{x_{1} \rightarrow x_{2}}<v_{x_{1}}^{i}(z), v_{x_{2}}^{j}(z)>_{\hat{F} \otimes S^{-}}
$$

Combining this with the identity obtained in Lemma 2.6 we find:

$$
\int_{\hat{T}^{4}} d z<u_{x}^{i}(z), u_{x}^{j}(z)>=\lim _{x_{1} \rightarrow x_{2}} \int_{\hat{T}^{4}} d z \exp \left(2 \pi i z\left(x_{1}-x_{2}\right)\right) \hat{d}^{*} \hat{d} G_{z}^{i j}\left(x_{1}, x_{2}\right) .
$$

From the definition of the Green's function one easily finds the short distance expansion:

$$
G_{z}^{i j}(x, y)=\frac{1}{4 \pi^{2}|x-y|^{2}}\left\{\delta_{i j}-\sum_{\mu}\left(A_{\mu}^{i j}(x)+2 \pi i z_{\mu} \delta_{i j}\right)\left(x_{\mu}-y_{\mu}\right)+\mathcal{O}\left(|x-y|^{2}\right)\right\}
$$


Partially integrating twice will give the desired result.

Collecting the results we have:

Theorem 2.8: $4 \pi \hat{\varepsilon}(G \Psi) \in \Gamma\left(T^{4} \times \hat{T}^{4}, \pi^{*} F^{*} \otimes \hat{\pi}^{*} \hat{F} \otimes \hat{\mathcal{P}} \otimes S^{-}\right)$gives a Hermitian isometry $F \rightarrow \hat{\hat{F}}$.

Theorem 2.9: Under the isomorphism of theorem 2.8, $A=\hat{\hat{A}}$.

Proof: By definition (see eqs.(1.5) and the discussion above Lemma 2.6)

$$
\begin{gathered}
\hat{\hat{A}}_{i j}^{\mu}=\int_{\hat{T}^{4}} d z<v_{x}^{i}(z), \frac{\partial v_{x}^{j}(z)}{\partial x_{\mu}}> \\
=\lim _{x_{1} \rightarrow x_{2}} \int_{\hat{T}^{4}} d z<v_{z}^{i}\left(x_{1}\right), \frac{\partial v_{z}^{j}\left(x_{2}\right)}{\partial x_{2}^{\mu}}>.
\end{gathered}
$$

Using Lemma 2.6 and equation (2.5), together with partially integrating twice will give the desired result.

In some sense it seems that the Nahm transfomation is perfect. It preserves self-duality, its square is the identity and as we shall see next; it gives a hyperKähler isometry between moduli spaces.

\section{Metric properties of Nahm's transform.}

We have seen that the Nahm transformation gives a diffeomorphism from the space $\mathcal{M}^{\prime}(F)$ of 1 -irreducible asd connections modulo gauge transformations to $\mathcal{M}^{\prime}(\hat{F})$. These spaces are open subsets of the moduli spaces $\mathcal{M}(F), \mathcal{M}(\hat{F})$ of instantons and it is well known that $\mathcal{M}(F), \mathcal{M}(\hat{F})$ are smooth manifolds away from the reducible connections (see Atiyah- Hitchin-Singer[3]). Moreover $\mathcal{M}(F)$ (and also of course $\mathcal{M}(\hat{F})$ ) are supplied with a Riemannian metric as follows. A tangent vector $X \in T_{A} \mathcal{M}^{\prime}$ can be uniquely represented by an element of $\Gamma\left(T^{4}, \Lambda^{1} \otimes\left(Q \times_{A d} u(n)\right)\right)$, also denoted by $X$, satisfying

$$
\begin{aligned}
d_{A}^{*} X=0 & \text { (Coulomb gauge condition) } \\
P_{+} d_{A} X=0 & \text { (deformation equation) }
\end{aligned}
$$

The $L^{2}$ - metric on $\Lambda^{1} \otimes\left(Q \times_{A d} u(n)\right)$ now induces a Riemannian metric on $\mathcal{M}(F)$. We shall show here that Nahm's transformation is an isometry with respect to this metric on the moduli spaces.

Suppose $a$ is a tangent vector to $A \in \mathcal{M}^{\prime}(F)$. The first step is to compute the infinitesimal change in the spinors, $\delta \psi_{z}^{j}(a)$. It is easily seen that $\delta \psi_{z}^{j}$ should satisfy $D_{A_{s}^{-}}^{-} \delta \psi_{z}^{j}=-a \cdot \psi_{z}^{j}$, where $a$ acts on $\psi_{z}^{j}$ by Clifford multiplication tensored with the fundamental representation of $U(\mathbf{n})$. One can normalize the $\delta \psi^{j}$ by requiring $\left\langle\delta \psi^{j}, \psi^{i}\right\rangle=0$, for all $i, j$. The normalized $\delta \psi^{j}$ is

$$
\delta \psi_{z}^{j}=-D_{A_{n}}^{+} G_{z}\left(a \cdot \psi_{z}^{j}\right)
$$

Combining this with equation (1.5) for the Nahm transformation, we obtain the following formula for the infinitesimal change $\hat{a} \in \Gamma\left(\hat{T}^{4}, \hat{\Lambda}^{1} \otimes\left(\hat{Q} \times_{A d} u(k)\right)\right)(k=\operatorname{rk}(\hat{F}))$, which is the derivative of the Nahm transformation applied to $a$ :

$$
\begin{aligned}
\hat{a}_{i j} & =-<D_{A_{x}}^{+} G_{z}\left(a \cdot \psi_{z}^{i}\right), \hat{d} \psi_{z}^{j}>-<\psi_{z}^{i}, \hat{d} D_{A_{z}}^{+} G_{z}\left(a \cdot \psi_{z}^{j}\right)> \\
& =2 \pi i<a \cdot \psi^{i}, G_{z}\left(d z^{k} e_{k} \cdot \psi_{z}^{j}\right)>+2 \pi i<d z^{k} e_{k} \cdot \psi^{i}, G_{z}\left(a \cdot \psi^{j}\right)> \\
& =<a \cdot \psi^{i}, \Omega \cdot G_{z} \psi_{z}^{j}>-<\Omega \cdot G_{z} \psi_{z}^{i}, a \cdot \psi_{z}^{j}>.
\end{aligned}
$$


The inner products are all with respect to $L^{2}\left(T^{4}, F \otimes S^{-}\right)$. The deformation of $\hat{A}$ could also be given by describing how the bundle $\hat{F}$ varies as a subbundle of $\hat{H} \rightarrow \hat{T}^{4}$, but this would not be a suitable description to compare metrics.

Proposition 3.1: If $A$ is asd and if $a$ satisfies the Coulomb gauge condition (3.1) then also $\hat{a}$, given by equation (3.3), satisfies the gauge condition.

Proof: Using the identities $\sum_{\mu} e_{\mu} D_{A_{s}}^{ \pm} e_{\mu}=2 D_{A_{z}}^{\mp}$ one finds easily that in the framing $\psi^{i}$

$$
\left(\hat{d}_{\hat{A}}^{*} \hat{a}\right)_{i j}=\sum_{\mu} 4 \pi^{2}<\psi_{z}^{i}, G_{z} \sum_{\mu} e_{\mu}\left(D_{A_{z}}^{-} \circ a-a \circ D_{A_{*}}^{+}\right) e_{\mu} \cdot G_{z} \psi_{z}^{j}>\in \Gamma\left(\hat{T}^{4}, T^{*} \hat{T}^{4} \otimes \text { End } \hat{E}\right)
$$

where $\circ$ denotes composition of operators. Now with $\phi \in \Gamma\left(T^{4}, S^{-} \otimes F\right)$

$$
\sum_{\mu} e_{\mu} \cdot D_{A_{z}}^{-}\left(a \cdot e_{\mu} \cdot \phi\right)-e_{\mu} \cdot a \cdot D_{A_{x}}^{+}\left(e_{\mu} \cdot \phi\right)=\sum_{\mu} e_{\mu} \cdot\left(D_{A_{x}}^{-} a\right) \cdot e_{\mu} \cdot \phi+2 \sum_{\mu, \alpha \neq \beta} e_{\mu} e_{\alpha} e_{\beta} e_{\mu} \cdot\left(a^{\beta} \nabla_{A_{x}}^{\alpha} \phi\right)
$$

where we substituted $D_{A_{z}}^{-}=e_{\mu} \nabla_{A_{x}}^{\mu}$ and $a=e_{\alpha} a^{\alpha}$. But $D_{A_{s}}^{-} a=0$ because the Dirac operator on 1-forms combines the deformation equation and the Coulomb gauge condition of equation (3.1), see Atiyah-HitchinSinger[3], §6. Finally it is a relation in the Clifford algebra of $R^{4}$ that: $\sum_{\mu} e_{\mu} e_{\alpha} e_{\beta} e_{\mu}=0$ if $\alpha \neq \beta$.

Proposition 3.2: The Nahm transformation preserves the $L^{2}-$ metric, provided the $u(n)$ metric is normalized as $\langle X, Y\rangle=-\operatorname{tr}(X Y)$.

Proof: Let $a \in T_{A} \mathcal{M}(F)$ and $\hat{a} \in T_{\hat{A}} \mathcal{M}(\hat{F})$ be arbitrary elements, satisfying equations (3.1) and its analogue for $\hat{F}$. We denote by $d N$ the derivative of the Nahm transformation $N: \mathcal{M}(F) \rightarrow \mathcal{M}(\hat{F})$, i.e. $d N(a)$ is given by equation (3.3). We have to show

$$
<d N(a), \hat{a}>_{\mathcal{M}(\hat{F})}=<a, d N(\hat{a})>_{\mathcal{M}(F)}
$$

since the right hand side equals $\left\langle a,(d N)^{-1}(\hat{\alpha})\right\rangle_{\mathcal{M}(F)}$ as $N^{2}=1$. Now equation (3.4) would follow from

$$
\begin{aligned}
& \int_{\hat{T}^{4}} d z<a \cdot \psi_{z}^{i}, 2 \pi i e_{\mu} \cdot G_{z} \psi_{z}^{j}>_{L^{2}\left(T^{4}, F \otimes S^{-}\right)} \hat{a}_{j i}^{\mu}= \\
& \qquad \int_{T^{4}} d x a_{\alpha \beta}^{\mu}<\hat{a} \cdot v_{x}^{\beta}, 2 \pi i e_{\mu} \cdot \hat{G}_{x} v_{x}^{\alpha}>_{L^{2}\left(\hat{T}^{4}, \hat{F} \otimes S^{-}\right)}
\end{aligned}
$$

where we used equtions (3.3) for the derivative of the Nahm transformation. The Green's function $\hat{G}_{x}$ is related to $\hat{G}$ in eqution $(2.3)$ by the gauge transformation $g(x, z)$ introduced in section $1: \hat{G}_{x}\left(z_{1}, z_{2}\right)=$ $\exp \left(-2 \pi i z_{1}(x)\right) \hat{G}\left(z_{1}, z_{2}\right) \exp \left(2 \pi i z_{2}(x)\right)$. Using now both the indices $i, j, \cdots$ for $\hat{F}$ and $\alpha, \beta, \cdots$ for $F$ we can rewrite equation (3.5), multiplying both sides with $-2 i$, as

$$
\int_{T^{4} \times \hat{T}^{*}} d x d z<a^{\alpha \beta} \cdot \psi_{\beta}^{i}, \hat{a}_{j i} \cdot \hat{\varepsilon}^{-1} u_{j}^{\alpha}>_{S^{-}}=\int_{T^{4} \times \hat{T}^{4}} d x d z<\hat{a}_{j i} \cdot u_{i}^{\alpha}, 4 \pi a_{\alpha \beta} \cdot\left(\hat{G} u^{\beta}\right)_{j}>_{S^{-}}
$$

or erasing the indices

$$
\int_{T^{4} \times \hat{T}^{4}}\left\langle\hat{a} u, a 4 \pi \hat{G} u>=\int_{T^{4} \times \hat{T}^{4}}\left\langle a \psi, \hat{a} \hat{\varepsilon}^{-1} u>\right.\right.
$$


Finally, and crucially, we make use of Lemma 2.4 which can be restated as

Lemma 3.3: $\Psi=\hat{\varepsilon}^{-1}(4 \pi \hat{G} u)$.

Proof: This follows directly from Lemma 2.4, Proposition 2.5 and the fact that $\hat{\varepsilon}^{-1} \hat{G} \hat{\varepsilon}=\hat{G}$.

Using this to rewrite the right hand side of equation (3.6) as

$$
\int_{T^{4} \times \hat{T}^{4}}<\hat{a}_{j i} \cdot u_{i}^{\alpha}, a_{\alpha \beta} \cdot \hat{\varepsilon} \psi_{\beta}^{j}>_{S^{-}},
$$

the fact that $\hat{\varepsilon}$ commutes with Clifford multiplication and that $\left\langle v, \hat{\varepsilon}^{-1} w\right\rangle=\langle w, \hat{\varepsilon} v\rangle$, one easily establishes equality with the lhs of equation (3.6)

The tangent bundle of $T^{4}$ can be equipped with a hyperKähler structure; that is there are three integrable complex structures $I, J, K$ on $T^{4}$ which satisfy quaternionic relations, and are all compatible with the orientation such that the metric is Kähler for each of them. It is a special feature of the anti-selfduality equations that also $\mathcal{M}(F)$ is a hyperKähler manifold where the complex structures on $T_{A} \mathcal{M}(F) \subset \Gamma\left(T^{4}, \Lambda^{1} \otimes\right.$ $\left.\left(Q \times_{A d} u(n)\right)\right)$ are simply given by those on $\Lambda^{1}$. We refer the reader to Atiyah-Hitchin[5], Mukai[17].

Theorem 3.4: $N: \mathcal{M}^{\prime}(F) \rightarrow \mathcal{M}^{\prime}(\hat{F})$ is a hyperKähler isometry.

Proof: It remains to show that the derivative $d N: a \rightarrow \hat{a}$ of Nahm's transformation commutes with a complex structure. This follows immediately from:

$$
\hat{a}_{i j}=\left\langle G_{z} \psi_{z}^{i}, \Omega \cdot a \cdot \psi_{z}^{j}>-<\Omega \cdot a \cdot \psi_{z}^{i}, G_{z} \psi_{z}^{j}>\right.
$$

which is equivalent to equation (3.3), but makes more explicit how $\Omega$ connects the two spaces of one-forms, in terms of which the complex stuctures are defined.

A. Maciocia pointed out to us that alternatively one can deduce the results of this section in the algebro-geometric setup as follows. Choosing a Kähler structure on $\mathcal{M}$ defines a complex analytic complex symplectic form on $\mathcal{M}$, see Mukai[17]. This form is the natural bilinear form on $H^{1}\left(T^{4}, \operatorname{End}_{0} F\right)$ with values in $H^{2}\left(T^{4}\right.$, End $\left.F\right)=C$. It can be shown that this pairing is preseved under the Fourier transform, thereby establishing that Nahm's transform is a Hyperkähler isometry. 


\section{References}

[1] Atiyah, M.F.: Geometry of Yang-Mills Fields, Fermi lectures, Scuola Normale Superiore, Pisa, 1979.

[2] Atiyah, M.F., Classical Groups and Classical Differential Operators on Manifolds: Differential Operators on Manifolds, CIME, Varenna (1975), 6-48.

[3] Atiyah, M.F., Hitchin, N.J., Singer, I.M., Selfduality in four-dimensional Riemannian Geometry, Proc. Roy. Soc. London, Ser. A, 362 (1978) 425-461.

[4] Atiyah, M.F., Singer, I.M., The Index of Elliptic Operators IV, Ann. of Math. 93 (1971) 119-138.

[5] Atiyah, M.F., Hitchin, N.J., The Geometry and Dynamics of Magnetic Monopoles, Porter Lectures, Princeton 1988.

[6] Corrigan, E., Goddard, P., Construction of Instanton and Monopole Solutions and Reciprocity, Ann. Phys. (N.Y.) 154 (1984) 253-279.

[7] Bismut, J.-M., Gillet, H., Soulé, C., Analytic Torsion and Holomorphic Determinant Bundles. III Quillen Metrics on Holomorphic Determinants, Comm. Math. Phys. 115 (1988) 301-351.

[8] Donaldson, S.K., Anti self-dual Yang-Mills Connections over Complex Algebraic Surfaces and Stable Vector Bundles, Proc. Lond. Math. Soc. (3) 50 (1985) 1-26.

[9] Donaldson, S.K., Nahm's Equations and the Classification of Monopoles, Comm. Math. Phys. 96 (1984) 387-407.

[10] Donaldson, S.K., Kronheimer, P.B., A Book on Yang-Mills Theory and 4-Manifolds, to appear Oxford, University Press.

[11] Donaldson, S.K., Connections, Cohomology and the Intersection Forms of 4-Manifolds, J. Diff. Geom. 24 (1986) 275-341.

[12] Duistermaat, J.J., Grünbaum, B., Differential Equations in the Spectral Parameter, Comm. Math. Phys. 103 (1986) 177.

[13] Hitchin, N.J., On the Construction of Monopoles, Comm. Math. Phys. 89 (1983) 145-190.

[14] 't Hooft, G., A property of Electric and Magnetic Flux in Non-Abelian Gauge Theories, Nucl. Phys. B153 (1979) 141.

[15] Hurtubise, J., Murray, M., The Moduli Space of SU(N) Monopoles and Nahm's Equations, Princeton preprint, 1988.

[16] Mukai, S., Duality between $D(X)$ and $D(\hat{X})$ with its Application to Picard Sheaves, Nagoya Math. J. 81 (1981) 153-175.

[17] Mukai, S., Symplectic Structure of the Moduli Space of Sheaves on an Abelian or K3 Surface, Inv. Math. 77 (1984) 101-116.

[18] Mukai, S., Fourier Functor and its Application to the Moduli of Bundles on an Abelian Variety, Adv. Studies in Pure Math. 10 (1987), Alg. Geometry Sendai, 1985, p.515-550.

[19] Nahm, W. Monopoles in Quantum Theory, In: Proceedings of the Monopole Meeting, Trieste, eds. Craigie, e.a., Singapore, World Scientific 1982. 
[20] Nahm, W., Self-Dual Monopoles and Calorons, In: Lect. Notes in Phys. 201, eds. Denardo, G., e.a., Springer-Verlag, Berlin, 1984.

[21] Schenk, H., On a Generalized Fourier Transform of Instantons over Flat Tori, Comm. Math. Phys. 116 (1988) $177-183$.

[22] Segal, G., Wilson, G., Loop Groups and the Equations of KdV type, Publ. Math. IHES 61, 1985.

[23] Taubes, C.H., Self-Dual Connections on 4-Manifolds with Indefinite Intersection Matrix, J. of Diff. Geom. 19 (1984) 517-560. 